\title{
Local transmission and global dissemination of New Delhi Metallo-Beta-Lactamase (NDM): a whole genome analysis
}

Wei Xin Khong ${ }^{1 \dagger}$, Eryu Xia ${ }^{2 \dagger}$, Kalisvar Marimuthu ${ }^{1,3+}$, Wenting Xu ${ }^{4}$, Yik-Ying Teo ${ }^{2,4,5,6,7}$, Eng Lee Tan ${ }^{8,9}$, Shiyong Neo ${ }^{8}$, Prabha Unny Krishnan ${ }^{10}$, Brenda S. P. Ang ${ }^{1}$, David C. B. Lye ${ }^{1,3}$, Angela L. P. Chow ${ }^{1,4}$, Rick Twee-Hee $\mathrm{Ong}^{4}$ and Oon Tek $\mathrm{Ng}^{1,4,11^{*}}$

\begin{abstract}
Background: New Delhi metallo- $\beta$-lactamase $\left(b / a_{N D M}\right)$, a plasmid-borne carbapenemase gene associated with significant mortality and severely limited treatment options, is of global public health concern as it is found in extremely diverse Gram-negative bacterial strains. This study thus aims to genetically characterize local and global spread of bla NDM.

Methods: To investigate local transmission patterns in the context of a single hospital, whole genome sequencing data of the first 11 bla $a_{\mathrm{NDM}}$-positive bacteria isolated in a local hospital were analyzed to: (1) identify and compare bla $a_{\text {NDM }}$-positive plasmids; and (2) study the phylogenetic relationship of the bacteria chromosomes. The global analysis was conducted by analyzing 2749 complete plasmid sequences (including

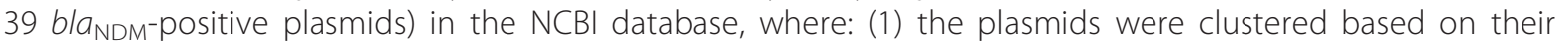
gene composition similarity; (2) phylogenetic study was conducted for each bla $a_{N D M}$-positive plasmid cluster to infer the phylogenetic relationship within each cluster; (3) gene transposition events introducing bla $a_{N D M}$ into different plasmid backbones were identified; and (4) clustering pattern was correlated with the plasmids' incompatibility group and geographical distribution.

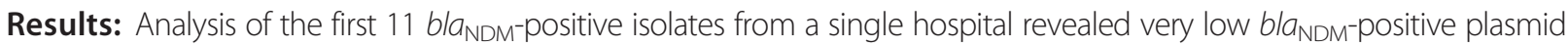
diversity. Local transmission was characterized by clonal spread of a predominant plasmid with 2 sporadic instances of plasmid introduction. In contrast to the low diversity locally, global bla $a_{\text {NDM }}$ spread involved marked plasmid diversity with no predominant bacterial clone. Thirty-nine (1.4\%) out of the 2749 complete plasmid sequences were bla $a_{\text {NDM-positive, }}$ and could be resolved into 7 clusters, which were associated with plasmid incompatibility group and geographical distribution. The bla NDM gene module was witnessed to mobilize between different plasmid backbones on at least 6 independent occasions.
\end{abstract}

Conclusions: Our analysis revealed the complex genetic pathways of bla NDM spread, with global dissemination characterized mainly by transposition of the bla $a_{\mathrm{NDM}}$ gene cassette into varied plasmids. Early local transmission following plasmid introduction is characterized by plasmid conjugation and bacterial spread. Our findings emphasize the importance of plasmid molecular epidemiology in understanding bla NDM $_{\text {spread. }}$

\footnotetext{
* Correspondence: oon_tek_NG@ttsh.com.sg

${ }^{\dagger}$ Equal contributors

${ }^{1}$ Institute of Infectious Disease and Epidemiology, Communicable Disease

Centre, Tan Tock Seng Hospital, Singapore, Singapore

${ }^{4}$ Saw Swee Hock School of Public Health, National University of Singapore,

Singapore, Singapore

Full list of author information is available at the end of the article
} 


\section{Background}

The emergence of carbapenemase-producing Enterobacteriaceae (CPE) has become an important threat to global health. CPE are primarily recognized in health care settings [1], with the prevalence from clinical samples increasing globally [2-6]. Outcomes of CPE infections are poor, where mortality associated with infections can reach over $40 \%[7,8]$. With the widespread dissemination of extended-spectrum $\beta$-lactamases, carbapenems are the last class of safe and effective antimicrobials for treating multidrug-resistant Gram-negative bacterial infections, the effectiveness of which has been greatly undermined by CPE [9]. As a result, there is a pressing need to understand the transmission pathways of carbapenemases to inform infection control, the main intervention available against $\mathrm{CPE}$ transmission and infection.

New Delhi metallo- $\beta$-lactamase $\left(b l a_{\mathrm{NDM}}\right)$ was first described in 2008 in a Swedish traveler returning from the Indian subcontinent [10]. Since then, $b l a_{\mathrm{NDM}}$ has been documented in all continents, with the earliest $b l a_{\mathrm{NDM}}$ archived sample from 2005 [11]. Compared with other carbapenemases, $b l a_{\mathrm{NDM}}$ spread is characterized by alarming public health features, including: (1) broad Gram-negative bacterial host range, including highlyvirulent bacteria such as Vibrio cholera and Shigella boydii [12]; (2) frequent acquisition among Escherichia coli and Klebsiella pneumoniae, which are Gram-negative species carried as gut flora and able to survive in the inanimate environment; (3) widespread presence in the Indian subcontinent, Southeast and East Asia, home to a large proportion of the global human population; and (4) co-carriage with other resistance genes on the bla $a_{\mathrm{NDM}}$-bearing plasmid [13].

Multiple seminal investigations have focused on determining international and local transmission patterns of chromosome-mediated antimicrobial resistance [14-16]. However, there remained many unanswered questions concerning the spread of plasmid-borne antimicrobial resistant genes. While mass global travel and widespread antibiotic use have been widely recognized as population risk factors associated with the dispersal of $b l a_{\text {NDM }}$ [13], little is known regarding the genomic factors associated with its rapid spread [17]. Antimicrobial resistance genes are often carried by mobile genetic elements like plasmids and transposons [18], which may also carry integrons or other gene mobilization elements $[19,20]$. A key biologic challenge in understanding plasmid-borne gene molecular epidemiology is its capability to exploit three tiers of gene spread: (1) inter-plasmid gene module transposition; (2) inter-bacterial plasmid conjugation; and (3) bacterial spread among humans, animals and the environment [13]. While single nucleotide polymorphism (SNP)-based phylogenetic methods have been successful in understanding the transmission of chromosomemediated antimicrobial resistance, these methods are illsuited to determining the dynamics of multi-tiered gene flow of plasmid-mediated antimicrobial resistance due to the lack of conserved genomic regions across the spectrum of diverse plasmids.

By moving beyond conventional SNP-based phylogenetic study to a plasmid clustering approach based on distances measured by the degree of gene sharing and similarity of shared genes between different plasmids, we analyzed a combined collection of all GenBank complete plasmid sequences within Gram-negative bacteria hosts, thus having an unprecedented opportunity to profile the global dissemination of this important resistance gene. A total of 2749 complete plasmid sequences from NCBI GenBank database were included in this study, of which 39 are $b l a_{\mathrm{NDM}}$-positive. This provided a comprehensive description on the distribution and genetic movement of $b l a_{\mathrm{NDM}}$. Moreover, in order to investigate local transmission of $b l a_{\mathrm{NDM}}$, we sequenced $11 b l a_{\mathrm{NDM}}$-positive $\mathrm{CPE}$ isolates in our institute [21], of which transmission pattern was inferred based on the identity of $b l a_{\mathrm{NDM}^{-}}$ positive plasmids and phylogenetic study of the chromosomes, in combination with the patients' records. In summary, our study suggested that $b l a_{\mathrm{NDM}}$-positive plasmid diversity is very low in a local Singapore hospital context. Analyzing the $11 b^{b} a_{\mathrm{NDM}^{-}}$-positive CPE isolated from the hospital showed that $b l a_{\mathrm{NDM}}$ spread was predominantly characterized by plasmid conjugation and bacterial transmission. In contrast, the $b l a_{\mathrm{NDM}}$-positive plasmid is highly variable in global setting, due to the transposition of the $b l a_{\mathrm{NDM}}$ gene cassette into different plasmids. Furthermore, our analysis revealed that bla $a_{\mathrm{NDM}}$-positive plasmids worldwide can be further clustered into 7 distinct clusters correlated with plasmid incompatibility group and geographical distribution. Our findings advance understanding of plasmid-mediated antimicrobial resistance spread both locally and globally.

\section{Methods \\ Clinical isolates}

Tan Tock Seng Hospital (TTSH) is Singapore's second largest acute-care hospital with 36 clinical and allied health departments and more than 1400 beds. The first case of carbapenemase-producing Enterobacteriaceae (CPE) in TTSH was detected in September 2010 (subject 16). Following the index case, CPE was detected in another 7 patients. Among these 7 patients, 2 shared a ward with the index patient during time at risk (ie, epidemiologically linked contacts), while the other 5 were detected via their clinical cultures. All the Enterobacteriaceae isolates from these CPE-positive patients were found to harbor $b l a_{\mathrm{NDM}}$. The infection control response to a new bla $a_{\mathrm{NDM}}$-positive patient detected in the course 
of routine testing included strict isolation of the index patient, contact tracing within the same ward and in previously admitted wards, and screening of these contacts with rectal swabs for CPE carriage using draft guidelines issued by CDC. Age, gender, travel history, history of ward locations and clinical diagnoses were collected by retrospective case-chart review.

In this hospital laboratory, species identification of Enterobacteriaceae was done using matrix-assisted laser desorption ionization-time of flight mass spectrometry (Bruker Daltoniks, Germany) and initial susceptibility testing was performed with VITEK 2 automated system (bioMérieux Vitek, Inc., USA) or using Kirby-Bauer method; susceptibility was defined according to the breakpoints of the Clinical and Laboratory Standard Institute (CLSI). Additional Minimum Inhibitory Concentration (MIC) testing using E-test strips (AB bioMerieux, Sweden) was done for all Enterobateriaceae isolates which were found to be non-susceptible to imipenem or meropenem on Mueller-Hinton plates; those isolates with a $\mathrm{MIC} \geq 2 \mathrm{mgs} / \mathrm{L}$ were investigated further. Characterization of $\beta$-lactamase genes was performed by a polymerase chain reaction (PCR) assay targeting three carbapenemases with potential for rapid spread: $b l a_{\mathrm{NDM}}$ 1 [22], $b l a_{\mathrm{KPC}}$ [23] and bla $a_{\mathrm{OXA}-48}$-like [24].

DNA sequencing and genome assembly of clinical isolates Whole genome sequencing was performed on the Illumina MiSeq platform (Illumina Inc., USA) as detailed before [21]. Sequence reads have been submitted to the European Nucleotide Archive (ENA) under accession PRJEB13304. De novo assembly was performed using Velvet [25], parameters of which were optimized by VelvetOptimiser (http://bioinformatics.net.au/software.velvetoptimiser.shtml) with $\mathrm{k}$-mer lengths ranging from 55 to 63. For all of the 11 isolates, VelvetOptimizer achieved the best assembly at the k-mer length of 63 .

Species identification for each isolate was performed using BLAST to search the assembled contigs in NCBI nt database. If the top five BLAST results for a contig are from chromosomal DNA, these chromosomal genomes would be considered as candidate chromosomes for the isolate. For each isolate, each candidate chromosomes would be used as the reference sequence, against which all the contigs would be aligned. The genome coverage by the contigs would then be calculated, where the candidate chromosome with the highest genome coverage would be taken as the most similar bacterial strain and its species would be identified as the bacterial species of the isolate. Multi-locus sequence type (MLST) databases for E. coli (Achtman scheme) and K. pneumonia were downloaded from pubmlst.org [26], BMC Bioinformatics and the assembled contigs were search against the gene alleles to infer MLST.

\section{Molecular epidemiology}

Sequence reads were aligned to the reference genome (JJ1886 [GenBank: CP006784.1] for E. coli, and HS11286 [GenBank: CP003200.1] for K. pneumoniae) using BWA [27]. Single-nucleotide variants were called using SAMtools [28]. Positions with less than 10 reads or with a minor allele frequency larger than 0.25 would be marked as 'unknown' data. Variants would then be called if the alternate allele frequency is above 0.75 . Maximum likelihood phylogenetic trees were constructed using RAxML [29] where a substitution model of GTRGAMMA was used and rapid bootstrap analysis was conducted on 500 runs. Neighbor-Joining trees were constructed using MEGA6 [30], where evolutionary distances were computed using the Kimura 2-parameter method and bootstrap test was conducted on 500 replicates.

\section{bla $a_{\mathrm{NDM}}$-positive plasmid identification}

For each isolate, the contig with the $b l a_{\mathrm{NDM}}$ gene was first identified and extracted, after which the contig sequence was searched in the NCBI nt database for complete plasmid sequences with more than 2000 basepair identity. The similar complete plasmid sequences were then each used as the reference genome, against which all the contigs were aligned to calculate the sequence coverage by the contigs. The complete sequences with the highest sequence coverage would then be taken as the most similar plasmids.

\section{Plasmid mapping, genome coverage calculation and variant calling}

Novoalign was used for reads mapping against a reference plasmid sequence, after which indel realignment was conducted with GATK IndelRealigner [31], and the coverage was calculated with GATK DepthOfCoverage. Variants were called with UnifiedGenotyper in GATK, with filtering criteria: "MQ $<40.0, \mathrm{QD}<2.0, \mathrm{FS}>60.0$, HaplotypeScore > 13.0".

\section{Conjugation assay}

Conjugation assays were performed using each of the 11 clinical isolates and an azide-resistant E. coli 553 as previously described [32]. To test the possibility of in vivo horizontal gene transfer in subject 21 , conjugation assay was repeated by mixing donor $K$. pneumoniae isolates (KP-U-141010 and KP-R-141010) with the recipient strain (EC-R-141010). E. coli trans-conjugants were selected using selective agar (Brilliance UTI Agar, Eosin methylene blue agar) supplemented with meropenem (10 mg/L). 


\section{Complete plasmid sequences}

All the 2749 available complete plasmid sequences within Gram-negative bacterial host in the NCBI plasmid database (April 2014) were downloaded for analysis, of which 39 are $b l a_{\mathrm{NDM}^{-}}$-positive. Information on sampling location and date, sample source, subject's travel history, host bacterial species and bacterial antimicrobial resistance phenotypes were obtained from GenBank entries or accompanying peer-reviewed references cited in GenBank.

\section{Plasmid clustering}

The plasmid clustering was conducted based on the virtual hybridization method as described by Zhou et al. [33] to investigate the similarity of the diverse complete plasmid sequences.

For each plasmid, all coding DNA sequences, as determined by original investigators, were downloaded from NCBI. Duplicate genes on the same plasmid, defined as coding DNA sequences having similarity value [similarity value $=$ length of matching sequences)*(BLAST identity)/(length of reference sequence)] above 0.45 , were removed. This resulted in a set of 234,450 genes. Additionally, insertion sequences within each plasmid were detected using IS Finder (https://www-is.biotoul.fr/) with default parameters at a cut-off e-value of $1 \mathrm{e}^{-20}$, which identified 1496 unique insertion sequences.

For genetic sequence comparison, a similarity score is calculated as $2 *$ (length of matching sequences)*(BLAST identity)/(length of reference sequence + length of matching sequences). The 2749 complete plasmid sequences were then compared using nucleotide BLAST algorithm against each of the 234,450 genes and 1496 insertion sequences to calculate a similarity score, which resulted in a 2749 by 235,946 matrix of similarity scores. A hypothetical plasmid sequence with all similarity scores set to zero was used as outgroup.

To achieve computational tractability, 1000 random matrices were generated, each of which was composed of $20 \%$ of the similarity score matrix's columns that were randomly selected without replacement, showing the similarity scores represented by the $20 \%$ of the randomly selected genes or insertion sequences. For each matrix of similarity scores, pair-wise Euclidean distances between plasmid sequences were calculated and formulated into a distance matrix, after which a NeighborJoining tree was constructed with the 'neighbor' program in PHYLIP [34]. A consensus tree was constructed using the 'consense' program in PHYLIP with the majority rule as the consensus type.

$b l a_{\mathrm{NDM}}$-positive plasmid clusters based on the consensus tree were defined using a stringent criterion of at least 2 unique $b l a_{\mathrm{NDM}}$-positive plasmids, with all nodes having $\geq 99 \%$ support at 1000 bootstraps.

\section{Phylogenetic tree for cluster refinement}

Cluster refinement was conducted for each cluster respectively. For each cluster, coding DNA sequences present in all plasmid sequences with a nucleotide BLAST e-value less than $1 \mathrm{e}^{-5}$ and an identity above $80 \%$ were extracted, aligned and concatenated. The phylogenetic trees were built for each cluster similarly as in the molecular epidemiology study.

\section{Incompatibility groups of plasmids}

Plasmid incompatibility (Inc) groups were assessed using PlasmidFinder from the Center for Genomic Epidemiology (CGE) with default settings [35].

\section{Comparative genomics}

To compare the plasmids, complete plasmid sequences and their corresponding gene features were first downloaded from NCBI Genbank. The annotated plasmid sequences were then compared and visualized with the Artemis comparison tool ACT [36].

\section{Results \\ Local bla $a_{\mathrm{NDM}}$-positive plasmid diversity in a single hospital}

The first 11 CPE isolates from 8 patients in a single Singapore hospital were isolated, of which the patient demographics and sample features are summarized in Table 1 and Fig. 1. The median duration of hospitalization to positive CPE culture was 3 days (range: 1-153 days). Six patients (subjects 16, 11, 1, 41, 51 and 53) had bla $a_{\text {NDM }}$ detected on clinical cultures. One patient (subject 21) was co-infected with 4 CPE isolates, where 2 different strains of Enterobacteriaceae were isolated from the patient's stool and urine samples, respectively. Of the 8 patients, only two had travelled out of Singapore in the past 2 years, including subject 21, who had travelled to Australia and subject 41, who had travelled to Malaysia. Whole genome sequencing was conducted on Illumina MiSeq, with the sequencing statistics summarized in Additional file 1: Table S1.

Plasmid identification was conducted with de novo assembly in combination with $b l a_{\mathrm{NDM}}$-positive plasmid identification, plasmid mapping and genome coverage calculation as elaborated in the Methods. The de novo assembly statistics were summarized in Additional file 1: Table S1. Among the 11 samples, 10 bla $a_{\mathrm{NDM}}$-positive plasmids were identified, of which 8 were identified as pTR3 [GenBank:JQ349086], 1 was identified as pNDMKN [GenBank:NC_019153] with the last being identified as pNDM_MGR194 [GenBank:NC_022740] (Table 1). Plasmid identification was most confident for the 41,187 base-pair plasmid pTR3 (100\% genome coverage in all the 8 identified samples at very high read depths) and the 46,253 base-pair plasmid pNDM_MGR194 (100\% 
Table 1 Patient demographics and sample features

\begin{tabular}{|c|c|c|c|c|c|c|}
\hline Subject ID & Travel history & Clinical diagnosis & Sample ID & Rationale for sample & $\begin{array}{l}\text { Identity of NDM } \\
\text { encoding plasmid }\end{array}$ & MLST \\
\hline 16 & NA & Colonization & EN-U-060910 & Clinical Sample & pTR3 & NA \\
\hline 11 & NA & Disease & KP-U-090910 & Clinical Sample & $\mathrm{pNDM}-\mathrm{KN}^{\mathrm{a}}$ & 437 \\
\hline 1 & NA & Colonization & EC-U-220910 & Clinical Sample & pTR3 & 410 \\
\hline \multirow[t]{4}{*}{21} & Australia & Colonization & KP-U-141010 & Clinical Sample & pTR3 & 48 \\
\hline & & Colonization & KP-R-141010 & Clinical Sample & pTR3 & 48 \\
\hline & & Colonization & EC-U-141010 & Clinical Sample & NA & 69 \\
\hline & & Colonization & EC-R-141010 & Clinical Sample & pTR3 & 69 \\
\hline 41 & Malaysia & Colonization & EC-U-101210 & Clinical Sample & pTR3 & 131 \\
\hline 46 & NA & Colonization & EC-R-141210 & $\begin{array}{l}\text { Contact Screening } \\
\text { for Index Subject } 41\end{array}$ & pTR3 & 131 \\
\hline 51 & NA & Disease & EC-B-220911 & Clinical Sample & pNDM_MGR194 & 205 \\
\hline 53 & NA & Disease & EC-U-191011 & Clinical Sample & pTR3 & 131 \\
\hline
\end{tabular}

Sample ID format: Organism- Specimen site-Date of Isolation (DD/MM/YY)

Organism: $\mathrm{EC}=$ Escherichia coli, $\mathrm{KP}=$ Klebsiella pneumoniae, $\mathrm{EN}=$ Enterobacter cloacae

Specimen site: $\mathrm{U}=$ Urine, $\mathrm{R}=$ Rectal swab, $\mathrm{B}=$ Bile

${ }^{a}$ Closest reference plasmid identified based on minimum $75 \%$ reference sequence coverage

genome coverage in sample EC- B-220911 at reasonable read depths). The 162,746 base-pair plasmid pNDMKN was identified in sample KP-U-090910 with $76.3 \%$ genome coverage at very high read depths. No

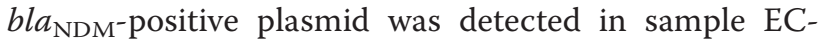
U-141010. The genome coverages and the read depths were summarized in Additional file 1: Figure S1 and Table S2, respectively.

Variant calling was performed for the 8 samples containing pTR3, the most prevalent $b l a_{\mathrm{NDM}}$-positive plasmid, to compare the pTR3 plasmid sequences in respective samples with the reference pTR3 sequence [GenBank:JQ349086]. Inspection of the variants revealed that 7 pTR3 plasmid sequences were identical to the reference pTR3 sequence, while one pTR3 plasmid sequence had only one SNP compared to the reference pTR3 sequence. In EN-U-060910 (isolated from subject 16), the pTR3 sequence had one synonymous mutation at the coding region of a putative transposase (position 22107), resulting a codon change of $\mathrm{GCC} \rightarrow \mathrm{GCT}$.

These results showed that local $b l a_{\mathrm{NDM}}$-positive plasmids had limited diversity with the majority of the plasmids being identical copies of pTR3, which is a strong indication of clonal plasmid spread. The other two bla $a_{\mathrm{NDM}}$-positive plasmids had identities of pNDM-KN and pNDM_MGR194. The major difference between the three plasmids (pTR3, pNDM-KN and pNDM_MGR194) strongly indicated independent plasmid introductions into the hospital ecology.

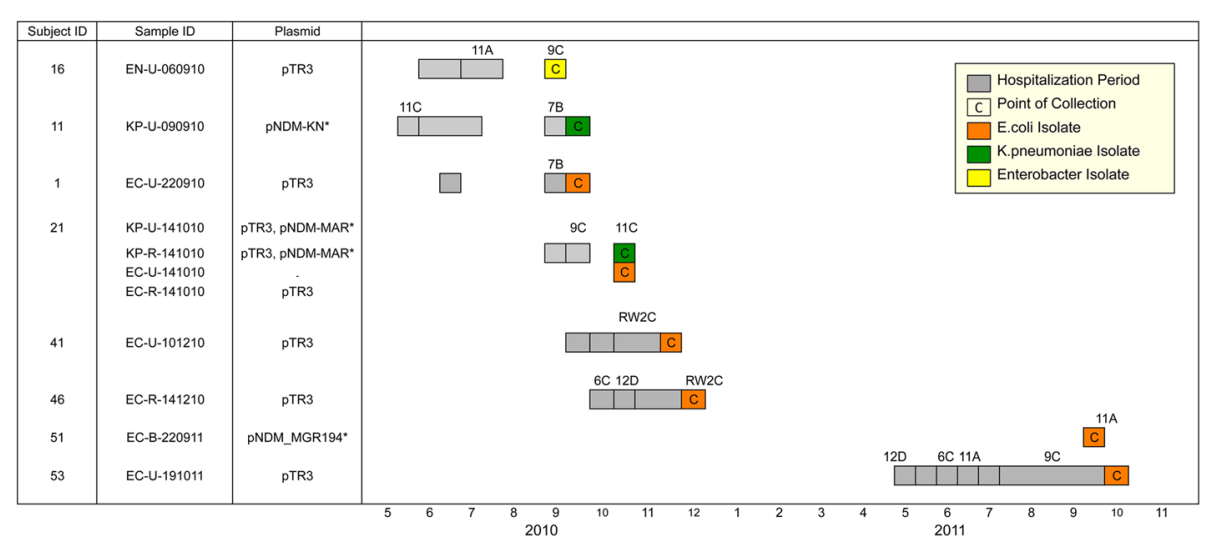

Fig. 1 Patient transmission dynamics of local bacteria samples. The bla $a_{N D M}$ cases were identified in a local hospital from 2010 to 2011 as represented in the timeline. Each patient is represented by a horizontal track. Subject ID, sample ID and bla $a_{\mathrm{NDM}}$-positive plasmid found in the isolate are indicated in the first 3 columns. Patient's stay in the same ward is denoted by gray box. Only wards with $\geq 2$ reported bla $a_{\text {NDM }}$ cases are indicated in the diagram. *: closest reference plasmid identified based on minimum $75 \%$ sequence coverage 


\section{Bacterial host range at the local level}

The bacterial species harboring $b l a_{\mathrm{NDM}}$-positive plasmids were: E. coli (7/11), K. pneumoniae (3/11) and Enterobacter cloacae (E.cloacae, 1/11) (Table 1). Of the $7 E$. coli isolates, 3 were most similar to ST131 E. coli strain NA114 [GenBank:NC_017644], while the remaining isolates were most similar to ST23 E. coli strain APEC O78 [GenBank:NC_020163], ST597 strain UMN026 [GenBank:NC_011751] and ST1128 strain IAI1 [GenBank:NC_011741]. For the K. pneumoniae isolates, three $K$. pneumoniae strains were identified to be similar, including: ST11 strain HS11286 [GenBank:NC_016845], ST23 strain NTUH-K2044 [GenBank:NC_012731] and ST23 strain 1084 [GenBank:NC_018522]. Consistent with previous report [37], there appeared to be no evidence of association between Enterobacteriaceae host species and specific plasmid identities.

Maximum likelihood phylogenetic trees were constructed for bacteria chromosomes respectively for $E$. coli (Fig. 2a) and K. pneumoniae (Fig. 2b), and were supported by Neighbor-Joining trees in Additional file 1:

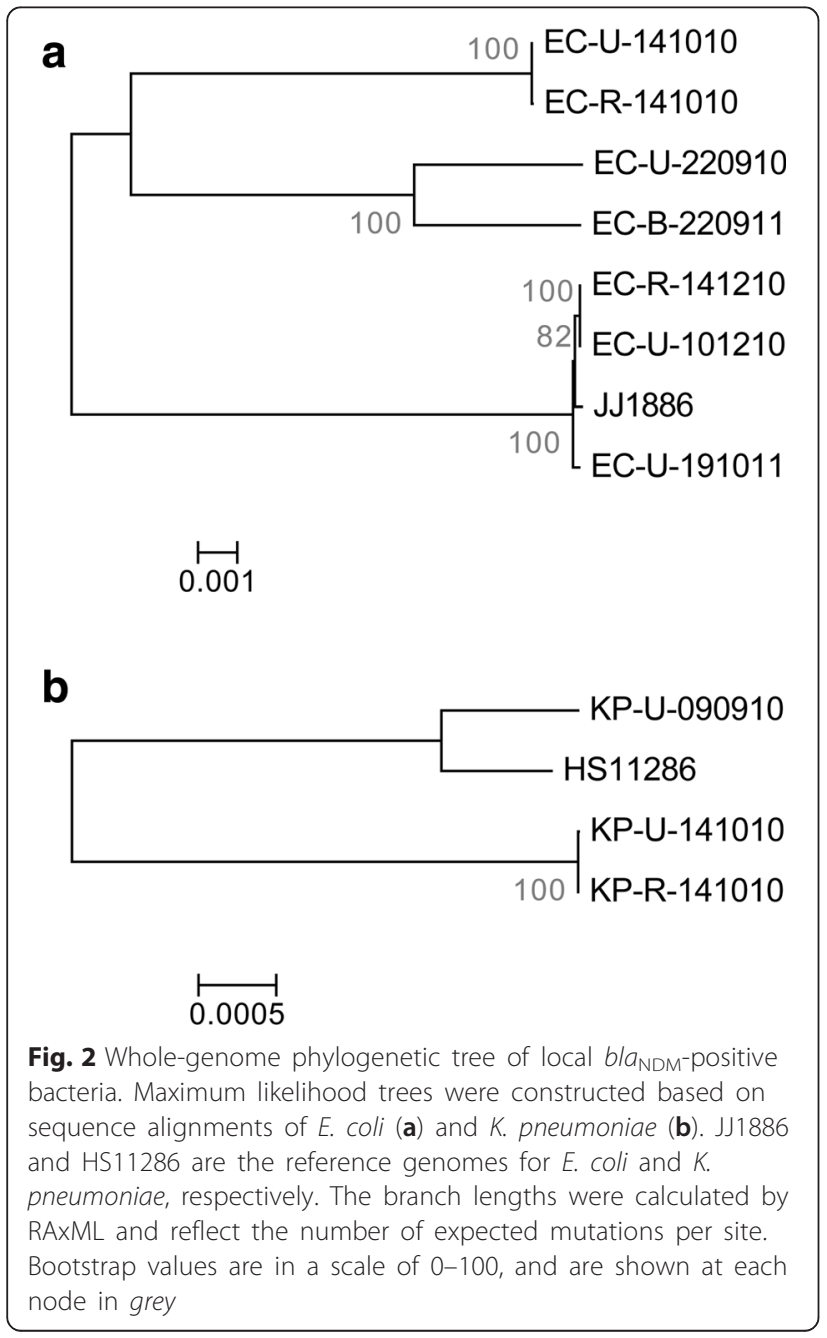

Figure S2. Phylogenetic trees for both E. coli and K. pneumoniae displayed significant diversity. The diversity of bacterial strains harboring pTR3 highlighted the propensity of $b l a_{\mathrm{NDM}}$-positive plasmids to spread via interbacterial plasmid conjugation, and would explain a key challenge in relying upon bacterial phylogenetic analysis alone to understand $b l a_{\mathrm{NDM}}$ dissemination.

Inter- and intra- patient bacterial spread at the local level Phylogenetic trees of the bacteria chromosomes in Fig. 2 suggested clonal bacterial spread in 3 instances. The first instance involved ST131 E. coli detected in 2 patients subjects 41 and 46, which clustered tightly as EC-U101210 and EC-R-141210 in Fig. 2a and differs by only 4 SNPs. The limited number of SNPs identified thereby suggested inter-patient bacterial spread.

The other two instances involved bacteria with identical sequence types isolated from different body sites in the same patient (subject 21). KP-U-141010 (isolated from urine) and KP-R-141010 (isolated from rectal swab) were both ST48 K. pneumoniae that harbored the pTR3 plasmid, which clustered tightly in Fig. $2 \mathrm{~b}$ with 25 SNPs. EC-U-141010 (isolated from urine) and EC-R141010 (isolated from rectal swab) are both ST69 E. coli that clustered tightly in Fig. 2a with 58 SNPs. Sample

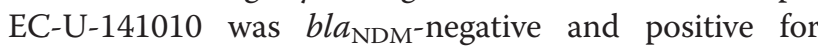

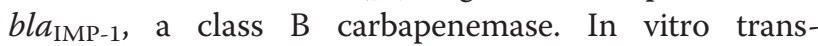
conjugation assays demonstrated the ability of pTR3 to conjugate from $K$. pneumoniae to $E$. coli. Mating assays with pTR3-positive $K$. pneumoniae from subject 21 as the donor and azide-resistant E. coli $\mathrm{J} 53$ as the recipient demonstrated pTR3 plasmid was self-conjugative (Additional file 1: Figure S3). Subject 21, as a result, represents a possible case of intra-host conjugation.

As discussed, the pTR3 plasmids remained $100 \%$ identical in all but 1 isolate at the nucleotide level in scenarios of inter- and intra-patient bacterial transfer, and inter-bacterial plasmid conjugation within the same host. These results suggested early spread of endemic plasmids at the local level was predominantly clonal.

\section{Clustering of global plasmids from Gram-negative bacterial} host

Complete genomic sequences of 2749 plasmids within Gram-negative bacterial hosts were downloaded from the NCBI database. The median plasmid sequence length is 30,949 base-pairs (range: 744-2,580,084), with the median number of genes annotated per plasmid being 36 (range: 1-2235). Out of the 2749 plasmids, the majority belong to the Enterobacteriaceae family $(n=$ 877, $31.9 \%)$, followed by Spirochaetaceae ( $n=405$, $14.7 \%)$, Rhodobacteraceae ( $n=85,3.1 \%)$, Moraxellaceae $(n=81,2.9 \%)$, and others $(n=1301,47.3 \%)$. 
Out of the 2749 plasmid sequences belonging to Gram-negative bacteria, 39 sequences were found to be $b l a_{\mathrm{NDM}}$-positive (Additional file 1: Table S3). These plasmids were sampled from all continents except Antarctica over an 8 year period (2005-2013). Thirtyeight of the 39 bla $a_{\mathrm{NDM}}$-positive plasmid samples have a human origin, while one sample has an environmental origin. The median plasmid sequence length for bla $_{\mathrm{NDM}}$-positive plasmids is 73,209 base-pairs (range: 35,947-288,920), with the median number of genes annotated per plasmid being 89 (range: 31372).

While construction of a SNP-based phylogenetic tree is the most common method to investigate evolutionary relationships among groups of organisms or strains, it is not applicable to plasmid phylogenetic study as there is no common genomic region shared among all the 2749 complete plasmid sequences. An alternative approach based on the relative distances measured by the degree of gene sharing and the similarity of shared genes has been applied to cluster the plasmids. The pair-wise distances based on a total of 234,450 genes and 1496 insertion sequences present in at least 1 plasmid were calculated as elaborated in the Methods, resulting in a Euclidean-distance derived distance matrix. A NeighborJoining tree was constructed with the distance matrix, upon which clustering analyses were based (Fig. 3). The clustering of global plasmid showed high global plasmid diversity with $b l a_{\mathrm{NDM}}$-positive plasmids located in different clusters.

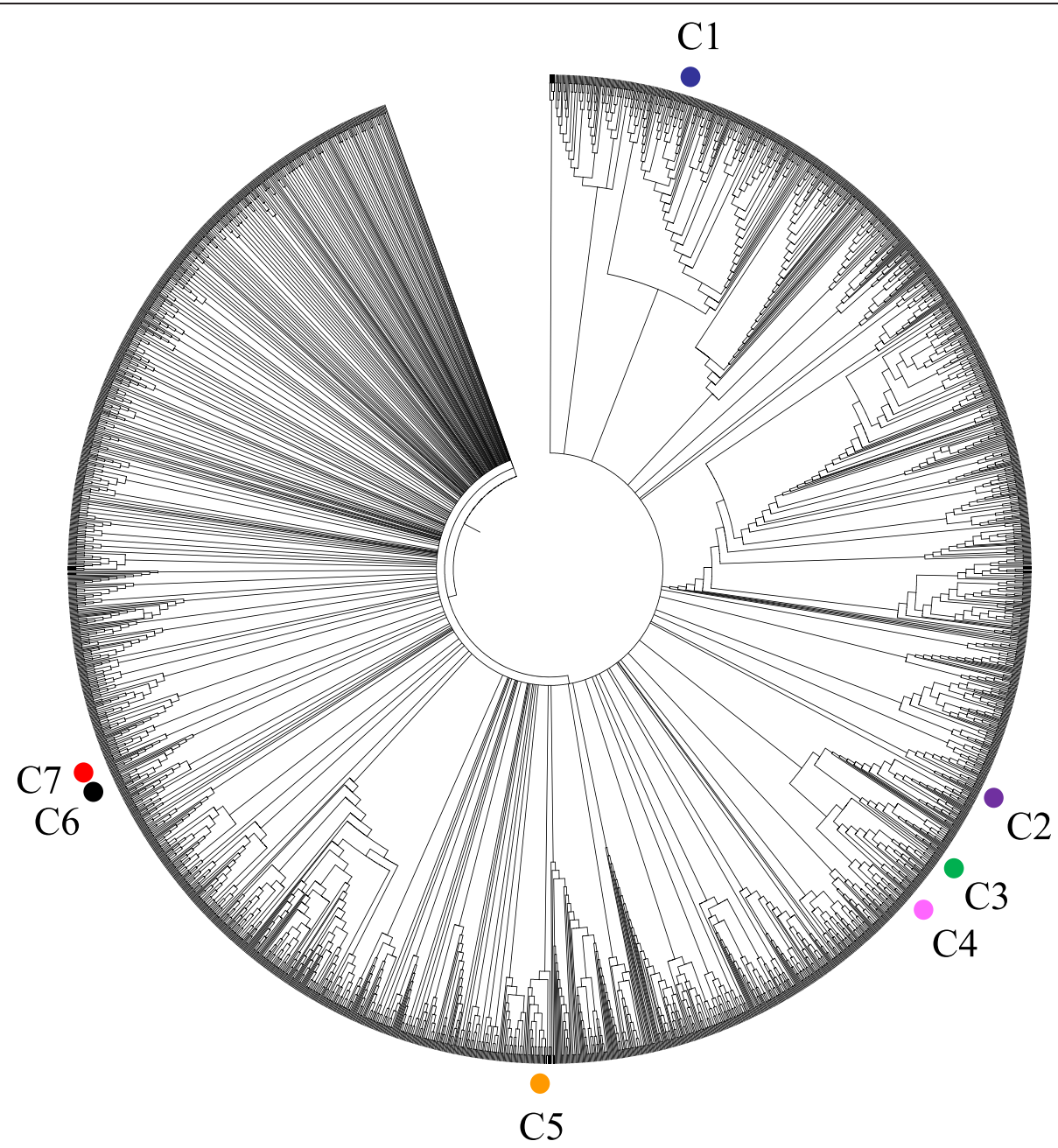

Fig. 3 Clustering of global plasmids in Gram-negative bacteria hosts. The Neighbor-Joining tree consisting of 2749 Gram-negative plasmid

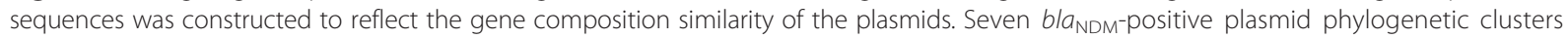

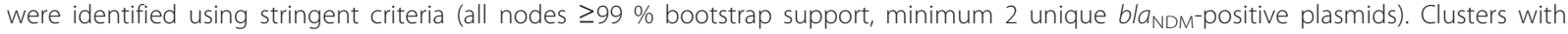
bla ${ }_{\mathrm{NDM}}$-positive plasmids are indicated with dots that are colored distinctively with blue (C1), purple (C2), green (C3), magenta (C4), orange (C5), grey (C6) and red (C7) 


\section{Clustering and phylogenetic study of $b / a_{\mathrm{NDM}}$-positive plasmids}

Seven clusters (represented by red dots in Fig. 3) were identified to contain $b l a_{\mathrm{NDM}}$-positive plasmids, which range in size from 2 to 10 plasmids. For better clarity, the plasmids within the seven clusters and the clusters near the seven clusters were extracted to construct a new Neighbor-Joining tree with the plasmids' information summarized (Additional file 1: Figure S4). The plasmids within the seven clusters were extracted and a new
Neighbor-Joining tree was constructed, which is presented as Fig. 4 with the plasmids' information.

The number of shared regions increased markedly for plasmids within the same cluster, allowing for the construction of a phylogenetic tree based on nucleotide sequence alignment within the shared regions. For clusters with more than three sequences, a concatenated alignment of the homologous sequences was generated, after which a phylogenetic tree would be constructed to study the phylogenetic relationship (Additional file 1: Figures

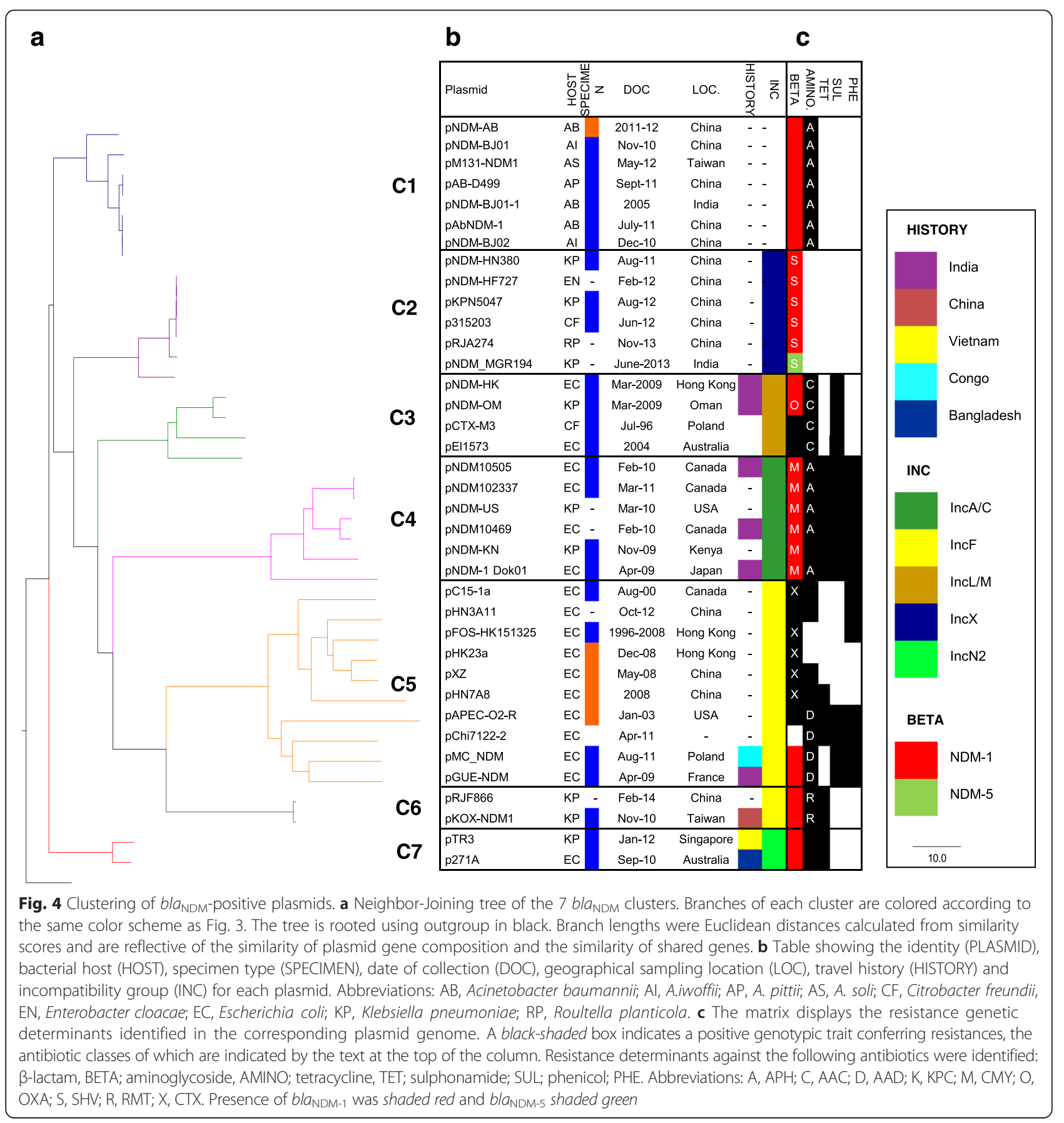


S5 and S6). The concatenated sequences within each cluster showed great similarity to each other, as can be identified by the short branch lengths.

While the distance-based clustering method provided a tree based on the gene composition similarity, the cluster refinement phylogenetic tree used SNPs to investigate the evolutionary relationship within each cluster, which were similar in topology with the clustering method.

\section{Global bla $a_{\text {NDM-positive plasmid diversity: gene }}$ transposition}

At least 6 events in the 7 clusters $(\mathrm{C} 1-\mathrm{C} 7)$ of $b a_{\mathrm{NDM}^{-}}$ positive plasmids have been observed to indicate independent recombination events introducing $b l a_{\mathrm{NDM}}$ into different plasmid backbones of $b l a_{\mathrm{NDM}}$-negative plasmids with subsequent evolution and spread (Fig. 5 and Additional file 1: Figure S7).

In the process of adaptive evolution, diversity of the microbial genome is primarily driven by recombination or point mutations $[38,39]$. As the clustering approach made use of plasmid gene composition diversity arising through recombination rather than point mutations, our findings suggested the $b l a_{\mathrm{NDM}}$-positive plasmids have undergone extensive mobile genetic element transposition to adapt to varying environmental niches. As mentioned earlier, there was minimal intra-cluster SNP difference, suggesting that polymorphisms due to point mutations play minimal role to account for the diversity of the plasmids.

Transpositions facilitated by transposons (Tn), insertion sequences (IS) elements and IS common region (ISCR) are detected frequently in plasmids that involve antimicrobial genes, non-antimicrobial genes and transposable genetic elements. With respect to the $b l a_{\mathrm{NDM}}$ gene, transposition mechanism involving bla $a_{\mathrm{NDM}}$ was discernible by comparative genomics in 4 instances: pNDM_HN380 [GenBank: JX104760] (C2, ISAba125mediated transposition, Fig. 5a), pNDM-OM [GenBank: JX988621] (C3, recombination into Tn1548-borne class I integrin, Fig. 5b), pEcNDM [GenBank: NC_023909] (unclustered, ISCR1-mediated transposition, Fig. 5c) and pNDM-BTR [GenBank: KF534788] (unclustered, fipA gene hotspot recombination, Fig. 5d). The Tn125 composite transposon platform has been theorized to be the original vehicle to mobilize bla ${ }_{\mathrm{NDM}}$ among Acinetobacter species. Our results reveal that $b l a_{\mathrm{NDM}}$ introductions also occurred in the context of ISCR1-mediated transposition, fipA gene hotspot recombination and Tn1548borne class I integron recombination. Larger datasets of genomic sequences involving $b l a_{\mathrm{NDM}}$-positive and negative nearest neighbors will enhance the understanding of $b l a_{\mathrm{NDM}}$ transposition globally.
Global bla $a_{\mathrm{NDM}}$-positive plasmid diversity: incompatibility group and geographical distribution

The plasmid clustering based on gene composition diversity tends to cluster the plasmids with the same backbone together, thus showing a clear clustering of the plasmid Inc groups for Enterobacteriaceae plasmids: plasmids in $\mathrm{C} 2$ are all Inc $\mathrm{X}$ plasmids, plasmids in $\mathrm{C} 3$ are Inc $\mathrm{L} / \mathrm{M}$, plasmids in $\mathrm{C} 4$ are $\mathrm{Inc} \mathrm{A} / \mathrm{C}$, plasmids in $\mathrm{C} 5$ and $\mathrm{C} 6$ are Inc F, while plasmids in $\mathrm{C} 7$ are Inc NII (Fig. 2).

The plasmid clusters also showed some association with geographical distributions. Some clusters were spreading mainly via regional transmission to date: (1) C1, a cluster of plasmids Acinetobacter sp. host, is limited to South Asia and East Asia; (2) C2 and C6 are limited to South and East Asia; and (3) C7 was found in Southeast Asia and Oceania. Other clusters (C3, C4 and C5) had wider geographic dispersion involving South Asia, East Asia, Middle East, North America, Africa and Europe.

\section{Local bla $a_{\mathrm{NDM}}$-positive plasmid in the global context}

As detailed in the global analysis, pTR3 clustered tightly with p271A [GenBank: JF785549], a plasmid described in Australia (Fig. 4, C7). The other two plasmids located in different plasmid clusters: pNDM-KN is in C4, and pNDM_MGR194 is in C2. In contrast to global plasmid diversity, the presence of near identical pTR3 plasmids in 8 out of 11 local samples suggested the $b l a_{\mathrm{NDM}^{-}}-$positive plasmid diversity at the local level to be very low. On the other hand, the 2 non-pTR3 plasmids, which were related to different plasmid clusters in the global plasmid phylogeny, were detected each in only one patient, which suggested independent plasmid introductions into the hospital ecology.

\section{Discussion}

By analyzing whole genome sequencing data of 11 bla $a_{\mathrm{NDM}}$-positive CPE isolated in a local hospital and 2749 complete plasmid sequences (including 39 bla $_{\mathrm{NDM}^{-}}$ positive plasmids) in the NCBI database, we investigated the local transmission and global dissemination of the $b l a_{\mathrm{NDM}}$ gene. Our analysis has highlighted the complex genetic pathways of $b l a_{\mathrm{NDM}}$ spread. Globally, $b l a_{\mathrm{NDM}}$ spread involved marked plasmid diversity with no predominant bacterial clone. The bla $a_{\mathrm{NDM}}$-positive plasmids were carried by multiple species of Acinetobacter and Enterobacteriaceae, thereby highlighting the propensity for conjugation of $b l a_{\mathrm{NDM}}$-positive plasmids among different bacterial species. The $b l a_{\mathrm{NDM}}$ gene module mobilized between different plasmid backbones on at least 6 independent occasions. In contrast to global plasmid diversity, early local spread of $b l a_{\mathrm{NDM}}$-positive plasmids in a single Singapore hospital was characterized by clonal 


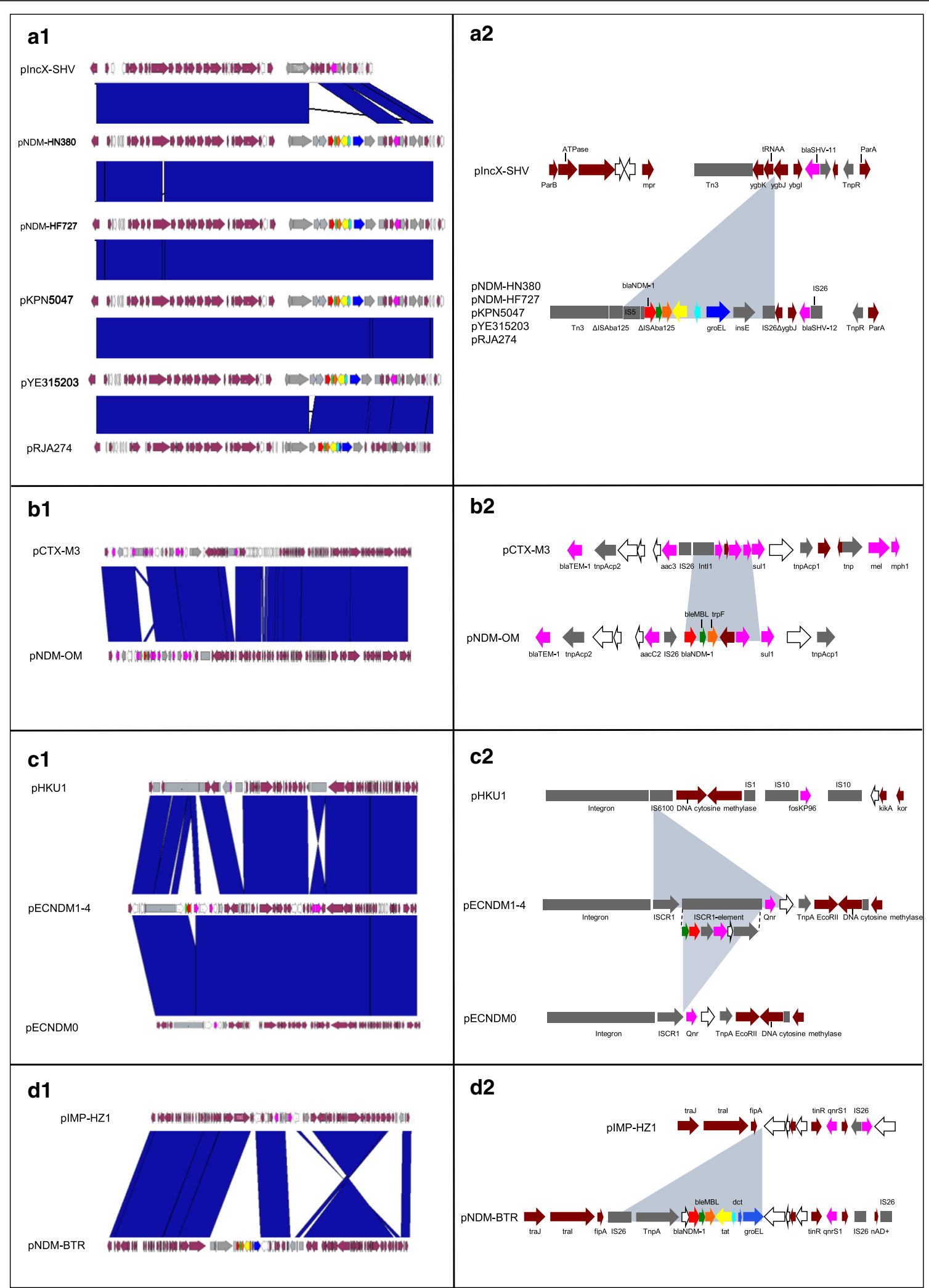

Fig. 5 (See legend on next page.) 
(See figure on previous page.)

Fig. 5 Acquisition of bla $a_{\mathrm{NDM}}$ cassettes. A1, B1, C1 and D1: A comparison of the bla $a_{\mathrm{NDM}}$-positive plasmid genomes with their putative backbone plasmids as identified in the plasmid clustering. The corresponding backbone plasmids are placed at the top of each column. Blue bands between panels indicate nucleotide BLAST matches with more than $99 \%$ sequence similarity. A2, B2, C2 and D2: Schematic representations of insertions in the bla NDM-positive plasmids (shaded in light blue) corresponding to A1, B1, C1 and D1. Annotated genes in these regions are colour coded. Arrows indicate predicted open-read frames (ORFs), genes with known functions (maroon), antimicrobial resistance genes (magenta), transpositional genetic elements (gray) and hypothetical proteins (white). Genes from the bla $a_{\text {NDM }}$ cassette are indicated by arrows coloured as follows: red, bla $a_{\text {NDM; }}$ green, ble MBLi orange, trpF; yellow, tat; light blue, dct; and dark blue, the groES- groEL cluster. Plasmid pECNDM represents an NDM-negative laboratory-derived plasmid, where the NDM cassette was mobilized from pECNDM1-4 as a free form

spread of a predominant plasmid pTR3 with 2 sporadic instances of plasmid introduction (pNDM-KN and pNDM_MGR194).

The plasmid clustering approach is crucial to our current analysis as it allows quantitative analyses of plasmid molecular epidemiology involving a large number of diverse plasmids as a tool in analyzing global spread of plasmid-borne genes. Prior genomic investigations of bla $a_{\mathrm{NDM}}$ spread have been mainly restricted to comparisons of less than 10 closely related plasmids due to the lack of phylogenetic congruence, and hence have not been able to discern the patterns of $b l a_{\mathrm{NDM}}$-positive plasmid clustering at a global level. The establishment of nearest-neighbor relationships facilitated the determination of transposition events involving genomic regions (genes and insertion sequences). Determination of cluster relationships subsequently opened the ability to correlate clusters with specific phenotypes (for example, extent of global spread or plasmid Inc groups).

Whole genome studies of successful bacterial clones have been used to understand transmission of chromosomallymediated antimicrobial resistant bacteria, for example MRSA. However, prior studies relying upon bacteria chromosomes to understand $b l a_{\mathrm{NDM}}$ transmission have been hindered by the diversity of bacterial species and strains harboring $b l a_{\mathrm{NDM}}$, even in a single geographic local [40]. The current study highlighted three vital evolutionary mechanisms underlying bla $a_{\mathrm{NDM}}$-positive bacteria diversity: (1) $b l a_{\mathrm{NDM}^{-}}$-gene module transposition, (2) bla $a_{\mathrm{NDM}}$-positive plasmid conjugation, and (3) bla $a_{\mathrm{NDM}}$-positive bacterial spread. Future studies of bla $a_{\mathrm{NDM}}$ transmission would have to take into account these three levels of gene spread.

Gene module transposition was a vital factor in the successful spread of $b l a_{\mathrm{NDM}}$ for at least three reasons: (1) mobilization of bla $a_{\mathrm{NDM}}$ from Acinetobacter $s p$. plasmids to Enterobacteriaceae plasmids, as recognized before; (2) mobilization of bla $a_{\mathrm{NDM}}$ among Enterobacteriaceae plasmids of differing Inc groups; and (3) nonbla $a_{\mathrm{NDM}}$ gene movement facilitating adaptation of plasmids to differing selection pressures.

It is well accepted that certain plasmid Inc groups can be stably maintained only in closely related bacterial hosts, while others have the ability to replicate in a broader host range [18]. In our study, we noted an association between plasmid Inc groups and geographical spread of the bla $a_{\mathrm{NDM}}$-positive plasmids. Specifically, broad-host-range (BHR) plasmid families (Inc A/C, Inc $\mathrm{L} / \mathrm{M}$ and Inc $\mathrm{F}$ ) were associated with transcontinental spread of $b l a_{\mathrm{NDM}}$ in Asia, North America, Africa and Europe. In contrast, narrow-host-range plasmids families (Inc X, Inc FII and Inc NII) had limited spread within the region. This suggested that Inc type contributes to the emergence and global spread of the bla $a_{\mathrm{NDM}}$ gene, as supported by multiple earlier studies. Laboratory experiments have showed that BHR plasmids evolved various strategies that enable them to replicate autonomously in much wider range of phylogenetically distinct hosts [18]. These plasmid types, being well adapted to different bacterial hosts (Inc $\mathrm{A} / \mathrm{C}$ and Inc $\mathrm{L} / \mathrm{M}$ ), endowed with efficient conjugative system (e.g Inc $\mathrm{L} / \mathrm{M}$ ) promoted the spread of $b l a_{\mathrm{NDM}}$ by enabling its stable maintenance in novel bacterial hosts or ecological niches, often independently from the presence of antimicrobial selection [18]. Collectively, our data suggested that plasmid types, especially those of BHR, play an essential role in plasmid adaptation by improving their stability for long-term persistence in bacterial communities of clinical and natural habitats. A limitation of the global plasmid-based inferences based on the current data is the limited number of samples and potential bias in sample selection, a limitation common for all studies leveraging on public databases. Larger studies with diverse sampling populations would help to address sampling bias.

Local bla $a_{\mathrm{NDM}}$ spread in a single Singapore hospital context was characterized predominantly by conjugation of a clonal plasmid (pTR3) between Enterobacteriaceae (inter-bacterial plasmid conjugation), and

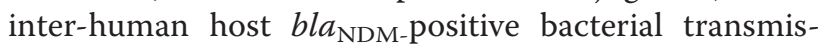
sion (bacterial spread). The finding of the pTR3 plasmid in 2 distinct $K$. pneumoniae strains in another Singapore hospital further supported a significant role of inter-human host transmission and clonal plasmid conjugation in local spread. Three recent publications using whole genome sequencing also reported the predominant role of inter-human host transmission (via the inanimate environment in some cases) and horizontal gene transfer in local hospital spread of carbapenemases [40-42]. 
One potential reason for the difference in the local and the global plasmid diversity is the sampling and the time period. While the 39 global complete $b l a_{\mathrm{NDM}}$-positive plasmid sequences has a long time range of eight years, the 11 local isolates were isolated within a one-year period.

An important limitation of this study and others of its kind is the inevitable bias that comes with selecting isolates. To minimize this inherent limitation, we included all $b l a_{\mathrm{NDM}}$ plasmid sequenced at the point of study, spanning a period of almost 8 years from 2005 to 2014 (note that $b l a_{\mathrm{NDM}}$ was only discovered in year 2009), covering 5 continents. Proportionally, a larger majority (85\%) of the samples came from human origin, while $b l a_{\mathrm{NDM}}$ has been reported to present in livestocks and environment in high prevalence. However, this would not inherently influence the results of this study, that is, linking phenotype (such as antimicrobial resistance, geographical location and host species) with plasmid characteristics (such as Inc group).

Our current analysis offers a glimpse of the genetic armamentarium available to $b l a_{\mathrm{NDM}}$ for dissemination to multiple environments. The limited data available for understanding transmission of this important resistance gene is highlighted by availability of only approximately

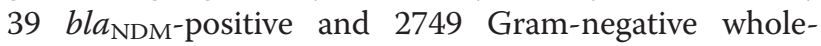
plasmid sequences globally. Whole genome sequencing of $b l a_{\mathrm{NDM}^{-}}$-positive isolates from diverse geographies on a much larger scale will definitely increase understanding of $b l a_{\mathrm{NDM}}$ evolution and spread, and may prove crucial to long-term control of NDM.

\section{Conclusions}

Our analysis has revealed the complex genetic pathways of $b l a_{\mathrm{NDM}}$ spread, where the global dissemination is mainly characterized by transposition of the $b l a_{\mathrm{NDM}}$ gene cassette into different plasmids while early local transmission is mainly a result of plasmid conjugation and bacterial spread. Our findings advance understanding of plasmid-mediated antimicrobial resistance spread both locally and globally.

\section{Abbreviations}

CPE, carbapenemase-producing Enterobacteriaceae; MLST, multi-locus sequence type; PCR, polymerase chain reaction; SNP, single nucleotide polymorphism.

\section{Additional file}

Additional file 1: Figure S1. Read depths along the reference plasmid sequences based on Illumina MiSeq sequencing reads mapping. Figure S2. Whole-genome Neighbor-Joining tree of local bla $\mathrm{NDM}^{-}$ positive bacteria. Figure S3. In vitro trans-conjugation assay of local bla $\mathrm{NDM}^{-}$

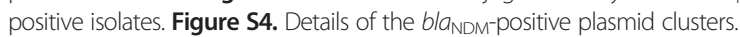
Figure S5. SNP-based refinement maximum likelihood trees of bla $a_{N D M}$ plasmid clusters. Figure S6. SNP-based refinement Neighbor-Joining trees of bla $a_{\text {NDM }}$ plasmid clusters. Figure S7. Investigation of transposition events among bla $a_{\text {NDM }}$ plasmid clusters by recombination analysis. Table S1. Summary of Illumina sequencing and de novo assembly statistics.

Table S2. Descriptive statistics for plasmid mapping. Table S3. Names and

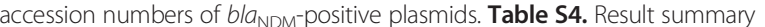
of recombination analysis. (DOCX $2781 \mathrm{~kb}$ )

\section{Acknowledgements}

We wish to thank all personnel who contributed to patient care and data collection for this analysis, Professor Jacoby for providing E. coli J53, and

Chua Hui Ying and Rosario Martinez Vega for their thoughtful review of the manuscript.

Funding

This study was supported by National Medical Research Council, Singapore (Grant No. TR12NMR047 and National Healthcare Group (Grant No.

TR14SIG006).

\section{Availability of data and material}

Sequence data were deposited in the European Nucleotide Archive (ENA) under accession PRJEB13304.

\section{Authors' contributions}

WXK, KM and OTN conceived the project. KM, PUK, BSPA, DCBL, OTN and ALPC collected the bacterial isolates and clinical information. WXK designed and performed the whole genome sequencing. EX, WXK, RTO, OTN, WX and YYT designed and performed the bioinformatics analysis. E.L.T and S.N performed the phenotypic assays. WXK, EX, RTO and OTN interpreted the results and wrote the manuscript. WXK, EX and KM contributed equally to the manuscript. All authors contributed and approved the final manuscript.

\section{Competing interests}

The authors declare that they have no competing interests.

\section{Consent for publication}

Not applicable.

\section{Ethics approval and consent to participate}

All methods were carried out in accordance with the approved guidelines. The study protocol was reviewed and approved by the Domain Specific Review Board, an ethics institutional review board of the National Healthcare Group in Singapore (ref no.: 2014/00535), which does not require that patients provide written informed consent. All authors vouch for the completeness and accuracy of the data and the analysis.

\section{Author details}

${ }^{1}$ Institute of Infectious Disease and Epidemiology, Communicable Disease Centre, Tan Tock Seng Hospital, Singapore, Singapore. ${ }^{2}$ NUS Graduate School for Integrative Science and Engineering, National University of Singapore, Singapore, Singapore. ${ }^{3}$ Yong Loo Lin School of Medicine, National University of Singapore, Singapore, Singapore. ${ }^{4}$ Saw Swee Hock School of Public Health, National University of Singapore, Singapore, Singapore. ${ }^{5}$ Life Sciences Institute, National University of Singapore, Singapore, Singapore. ${ }^{6}$ Department of Statistics and Applied Probability, National University of Singapore, Singapore, Singapore. ${ }^{7}$ Genome Institute of Singapore, Agency for Science, Technology and Research, Singapore, Singapore. ${ }^{8} \mathrm{Centre}$ for Biomedical and Life Sciences, Singapore Polytechnic, Singapore, Singapore. ${ }^{9}$ Department of Pediatrics, University Children's Medical Institute, National University of Singapore, Singapore, Singapore. ${ }^{10}$ Department of Laboratory Medicine, Tan Tock Seng Hospital, Singapore, Singapore. ${ }^{11}$ Communicable Disease Centre, Tan Tock Seng Hospital, 11, Jalan Tan Tock Seng, Singapore 308433, Singapore.

Received: 11 February 2016 Accepted: 14 May 2016

Published online: 13 June 2016

References

1. Perez F, Endimiani A, Ray AJ, Decker BK, Wallace CJ, Hujer KM, Ecker DJ, Adams MD, Toltzis P, Dul MJ, Windau A, Bajaksouzian S, Jacobs MR, Salata RA, Bonomo RA. Carbapenem-resistant Acinetobacter baumannii and Klebsiella pneumoniae 
across a hospital system: impact of post-acute care facilities on dissemination J Antimicrob Chemother. 2010;65:1807-18.

2. Hsu LY, TAN TY, Jureen R, Koh TH, Krishnan P, Tzer-Pin Lin R, Wen-Sin Tee N, Tambyah PA. Antimicrobial drug resistance in Singapore hospitals. Emerg Infect Dis. 2007;13:1944-7.

3. Xiao Y-H, Giske CG, Wei Z-Q, Shen P, Heddini A, Li L-J. Epidemiology and characteristics of antimicrobial resistance in China. Drug Resist Updat. 2011; 14:236-50.

4. van Duijn PJ, Dautzenberg MJD, Oostdijk EAN. Recent trends in antibiotic resistance in European ICUs. Curr Opin Crit Care. 2011;17:658-65.

5. Rhomberg PR, Jones RN. Summary trends for the Meropenem Yearly Susceptibility Test Information Collection Program: a 10-year experience in the United States (1999-2008). Diagn Microbiol Infect Dis. 2009;65:414-26.

6. Prabaker $\mathrm{K}$, Weinstein RA. Trends in antimicrobial resistance in intensive care units in the United States. Curr Opin Crit Care. 2011:17:472-9.

7. Schwaber MJ, Klarfeld-Lidji S, Navon-Venezia S, Schwartz D, Leavitt A, Carmeli Y. Predictors of carbapenem-resistant Klebsiella pneumoniae acquisition among hospitalized adults and effect of acquisition on mortality. Antimicrob Agents Chemother. 2008:52:1028-33.

8. Bratu S, Landman D, Haag R, Recco R, Eramo A, Alam M, Quale J. Rapid spread of carbapenem-resistant Klebsiella pneumoniae in New York City: a new threat to our antibiotic armamentarium. Arch Intern Med. 2005;165:1430-5.

9. Pitout JDD, Laupland KB. Extended-spectrum beta-lactamase-producing Enterobacteriaceae: an emerging public-health concern. Lancet Infect Dis. 2008:8:159-66.

10. Yong D, Toleman MA, Giske CG, Cho HS, Sundman K, Lee K, Walsh TR. Characterization of a new metallo-beta-lactamase gene, bla(NDM-1) and a novel erythromycin esterase gene carried on a unique genetic structure in Klebsiella pneumoniae sequence type 14 from India. Antimicrob Agents Chemother. 2009;53:5046-54.

11. Jones LS, Toleman MA, Weeks JL, Howe RA, Walsh TR, Kumarasamy KK. Plasmid carriage of bla $a_{\mathrm{NDM}-1}$ in clinical Acinetobacter baumannii isolates from India. Antimicrob Agents Chemother. 2014;58:4211-3.

12. Kumarasamy KK, Toleman MA, Walsh TR. Emergence of a new antibiotic resistance mechanism in India, Pakistan, and the UK: a molecular, biological, and epidemiological study. Lancet Infect Dis. 2010;10:597-602.

13. Wailan AM, Paterson DL. The spread and acquisition of NDM-1: a multifactorial problem. Expert Rev Anti Infect Ther. 2014;12:91-115.

14. Grad YH, Kirkcaldy RD, Trees D, Dordel J, Harris SR, Goldstein E, Weinstock H, Parkhill J, Hanage WP, Bentley S, Lipsitch M. Genomic epidemiology of Neisseria gonorrhoeae with reduced susceptibility to cefixime in the USA: a retrospective observational study. Lancet Infect Dis. 2014;14:220-6.

15. Harris SR, Clarke IN, Seth-Smith HMB, Solomon AW, Cutcliffe LT, Marsh P, Skilton RJ, Holland MJ, Mabey D, Peeling RW, Lewis DA, Spratt BG, Unemo M, Persson K, Bjartling C, Brunham R, de Vries HJC, Morré SA, Speksnijder A, Bébéar CM, Clerc M, de Barbeyrac B, Parkhill J, Thomson NR. Whole-genome analysis of diverse Chlamydia trachomatis strains identifies phylogenetic relationships masked by current clinical typing. Nat Genet. 2012:44:413-9. S1.

16. Harris SR, Feil EJ, Holden MTG, Quail MA, Nickerson EK, Chantratita N, Gardete S, Tavares A, Day N, Lindsay JA, Edgeworth JD, de Lencastre H, Parkhill J, Peacock SJ, Bentley SD. Evolution of MRSA during hospital transmission and intercontinental spread. Science. 2010:327:469-74.

17. Poirel L, Dortet L, Bernabeu S, Nordmann P. Genetic Features of bla $a_{\mathrm{NDM}-1^{-}}$ Positive Enterobacteriaceae. Antimicrob Agents Chemother. 2011;55:5403-7.

18. Carattoli A. Resistance plasmid families in Enterobacteriaceae. Antimicrob Agents Chemother. 2009;53:2227-38.

19. Cambray G, Guerout A-M, Mazel D. Integrons. Annu Rev Genet. 2010;44:141-66.

20. Schultsz C, Geerlings S. Plasmid-mediated resistance in Enterobacteriaceae: changing landscape and implications for therapy. Drugs. 2012;72:1-16.

21. Xia E, Khong WX, Marimuthu K, Xu W, Ong RT-H, Tan EL, Krishnan PU, Ang BSP, Lye DC, Chow ALP, Teo Y-Y, Ng OT. Draft genome sequence of a multidrug-resistant New Delhi Metallo- $\beta$-Lactamase-1 (NDM-1)-producing Escherichia coli isolate obtained in Singapore. Genome Announc. 2013;1: e01020-131.

22. Teo J, Ngan G, Balm M, Jureen R, Krishnan P, Lin R. Molecular characterization of NDM-1 producing Enterobacteriaceae isolates in Singapore hospitals. Western Pac Surveill Response J. 2012;3:19-24.

23. Balm MND, Ngan G, Jureen R, Lin RTP, Teo J. Molecular characterization of newly emerged bla $a_{\mathrm{KPC}-2}$-producing Klebsiella pneumoniae in Singapore. J Clin Microbiol. 2012;50:475-6.
24. Balm MND, Ngan G, Jureen R, Lin RTP, Teo JWP. OXA-181-producing Klebsiella pneumoniae establishing in Singapore. BMC Infect Dis. 2013;13:58.

25. Zerbino DR, Birney E. Velvet: algorithms for de novo short read assembly using de Bruijn graphs. Genome Res. 2008;18:821-9.

26. Jolley KA, Maiden MC. BIGSdb: Scalable analysis of bacterial genome variation at the population level. BMC Bioinformatics. 2010;11:595. doi:10.1186/1471-2105-11-595.

27. Li H, Durbin R. Fast and accurate long-read alignment with Burrows-Wheeler transform. Bioinformatics. 2010;26:589-95.

28. Li H, Handsaker B, Wysoker A, Fennell T, Ruan J, Homer N, Homer N, Marth G, Abecasis G, Durbin R, 1000 Genome Project Data Processing Subgroup. The sequence alignment/map format and SAMtools. Bioinformatics. 2009;25:2078-9.

29. Stamatakis A. RAxML version 8: a tool for phylogenetic analysis and postanalysis of large phylogenies. Bioinformatics. 2014;30:1312-3.

30. Tamura K, Stecher G, Peterson D, Filipski A, Kumar S. MEGA6: Molecular Evolutionary Genetics Analysis version 6.0. Mol Biol Evol. 2013;30:2725-9.

31. McKenna A, Hanna M, Banks E, Sivachenko A, Cibulskis K, Kernytsky A, Garimella K, Altshuler D, Gabriel S, Daly M, DePristo MA. The genome analysis toolkit: a MapReduce framework for analyzing next-generation DNA sequencing data. Genome Res. 2010;20:1297-303.

32. Ho PL, Li Z, Lo WU, Cheung Y-Y, Lin CH, Sham P-C, Chi-Chung Cheng V, $\mathrm{Ng}$ T-K, Que T-L, Chow KH. Identification and characterization of a novel incompatibility group X3 plasmid carrying bla NDM-1 $_{\text {in Enterobacteriaceae }}$ isolates with epidemiological links to multiple geographical areas in China. Emerg Microbes Infect. 2012:1:e39.

33. Zhou Y, Call DR, Broschat SL. Genetic relationships among 527 Gram-negative bacterial plasmids. Plasmid. 2012;68:133-41.

34. Rivas E, Eddy SR. Probabilistic phylogenetic inference with insertions and deletions. PLoS Comput Biol. 2008:4:e1000172

35. Carattoli A, Zankari E, Garcia-Fernandez A, Larsen MV, Lund O, Villa L, Aarestrup FM, Hasman H. In silico detection and typing of plasmids using PlasmidFinder and plasmid multilocus sequence typing. Antimicrob Agents Chemother. 2014 58:3895-903.

36. Carver TJ, Rutherford KM, Berriman M, Rajandream M-A, Barrell BG, Parkhill J. ACT: the Artemis Comparison Tool. Bioinformatics. 2005;21:3422-3.

37. Teo JWP, Tan P, La M-V, Krishnan P, Tee N, Koh TH, Deepak RN, Tan TY, Jureen R, Lin RTP. Surveillance trends of carbapenem-resistant Enterobacteriaceae from Singapore, 2010-2013. Integ Med Res. 2013;2:1-4.

38. Bryant J, Chewapreecha C, Bentley SD. Developing insights into the mechanisms of evolution of bacterial pathogens from whole-genome sequences. Future Microbiol. 2012;7:1283-96.

39. Croucher NJ, Harris SR, Fraser C, Quail MA, Burton J, van der Linden M, McGee L, Gottberg von A, Song JH, Ko KS, Pichon B, Baker S, Parry CM, Lambertsen LM, Shahinas D, Pillai DR, Mitchell TJ, Dougan G, Tomasz A, Klugman KP, Parkhill J, Hanage WP, Bentley SD. Rapid pneumococcal evolution in response to clinical interventions. Science. 2011;331:430-4.

40. Stoesser N, Giess A, Batty EM, Sheppard AE, Walker AS, Wilson DJ, Didelot X Bashir A, Sebra R, Kasarskis A, Sthapit B, Shakya M, Kelly D, Pollard AJ, Peto TEA, Crook DW, Donnelly P, Thorson S, Amatya P, Joshi S. Genome Sequencing of an Extended Series of NDM-Klebsiella pneumoniae Neonatal Infections in a Nepali Hospital Characterizes the Extent of Community Versus Hospital-associated Transmission in an Endemic Setting. Antimicrob Agents Chemother. 2014;58(12):7347-57.

41. Conlan S, Thomas PJ, Deming C, Park M, Lau AF, Dekker JP, Snitkin ES, Clark TA, Luong K, Song Y, Tsai Y-C, Boitano M, Dayal J, Brooks SY, Schmidt B, Young AC, Thomas JW, Bouffard GG, Blakesley RW, NISC Comparative Sequencing Program, Mullikin JC, Korlach J, Henderson DK, Frank KM, Palmore TN, Segre JA. Single-molecule sequencing to track plasmid diversity of hospital-associated carbapenemaseproducing Enterobacteriaceae. Sci Transl Med. 2014;6:254ra126.

42. Snitkin ES, Zelazny AM, Thomas PJ, Stock F, NISC Comparative Sequencing Program, Henderson DK, et al. Tracking a hospital outbreak of carbapenemresistant Klebsiella pneumoniae with whole-genome sequencing. Sci Transl Med. 2012:4:148ra116 